\title{
NEUROMODULACIÓN PERIFÉRICA EN EL TRATAMIENTO DE LA INCONTINENCIA DE ORINA: EFECTO DE LA ESTIMULACIÓN TRANSCUTÁNEA DEL NERVIO TIBIAL POSTERIOR SOBRE LA VEJIGA HIPERACTIVA
}

\author{
Paolo Ricci A. ${ }^{1}$, Odette Freundlich K. ${ }^{1 a}$, Vicente Solà D. ${ }^{1}$, Jack Pardo S. ${ }^{1}$ \\ 1 Unidad de Uroginecología, Departamento de Obstetricia y Ginecología, Clínica Las Condes.
}

a Kinesióloga

\section{RESUMEN}

Objetivo: Demostrar la efectividad de la neuromodulación con el sistema de estimulación transcutánea del nervio tibial posterior, en la inhibición de las contracciones no inhibidas del detrusor, en paciente con urgeincontinencia refractaria a tratamiento. Paciente: Se presenta un caso de incontinencia de orina mixta, con solución al componente de esfuerzo mediante TVT-Secur. El componente de urgencia, dado por vejiga hiperactiva, se trata mediante neuromodulación transcutánea del nervio tibial posterior, refractario al tratamiento medicamentoso y fisioterapia. Se demuestra objetivamente la acción de la neuromodulación, mediante la realización de una cistometría (urodinamia monocanal) durante la estimulación de nervio tibial (estímulos en modo continuo a $20 \mathrm{~Hz}$ y 200 milisegundos), comparada con la realizada sin estimulación. Resultados: Las contracciones no inhibidas del detrusor se inhibieron al realizar la neuromodulación con el sistema de estimulación transcutáneo del nervio tibial posterior, demostrado objetivamente por la desaparición de las contracciones durante la fase de llenado vesical. La capacidad cistométrica máxima, aumentó durante la neuromodulación y la sensación urgencia miccional disminuyó. Conclusión: La neuromodulación con el sistema de estimulación transcutánea del nervio tibial posterior, es efectiva en el tratamiento de la vejiga hiperactiva, lo que es posible de objetivar mediante la realización de una cistometría con urodinamia monocanal. Esta terapia ofrece una alternativa de tratamiento, con todas las características de mínima invasión.

\section{PALABRAS CLAVE: Incontinencia de orina, detrusor hiperactivo, vejiga hiperactiva, urge-inconti- nencia, urodinamia}

\section{SUMMARY}

Objective: To demonstrate objectively the efficiency of the neuromodulation with the transcuteneous posterior tibial nerve stimulation in overactive bladder, in a patient with urge incontinence, refractory to traditional treatments. Patient: A case of urinary mixed incontinence is presented. The TVT-Secur was used for the stress urinary incontinence. The urge-incontinence due to overactive bladder was treated by transcutaneous tibial nerve neuromodulation when refractory appearing to the treatment with medicines and physiotherapy by thirty days. The action of the neuromodulation is demonstrated objectively by cystometry. Monochannel urodynamic equipment was used. The Biomed 2000 equipment was used for the transcutaneous neuromodulation, with stimuli in continuos way to $20 \mathrm{Hertz}$ and 200 milliseconds. Results: The transcutaneous tibial 
nerve neuromodulation is able to inhibit the contraction of the detrusor, in a patient with urge-incontinence refractory to medical treatment. This is demonstrated objectively by the disappearance of the contraction noninhibited of the detrusor in the uridinamic cistometry. The maximum cystometric capacity increases and the urge-incontinence sensation diminish. Conclusion: The tibial nerve transcutaneous stimulation is an effective treatment in the overactive bladder, and the objective acute effect can be observed on the urodynamic cystometry. This therapy corresponds to a noninvasive treatment.

\section{KEY WORDS: Urinary incontinence, overactive detrusor, overactive bladder, urge incontinence, urodynamic}

\section{INTRODUCCIÓN}

La vejiga hiperactiva (VH) es una condición caracterizada por urgencia miccional, que puede expresarse con o sin urge-incontinencia, y usualmente con aumento de la frecuencia miccional y nicturia, condición que se asocia con aumento de los riesgos de afectar la salud $(1,2)$.

Un estudio realizado en EEUU, en 5204 adultos mayores de 18 años, estimó que $16 \%$ de los varones y $16,9 \%$ de las mujeres, padece de vejiga hiperactiva con o sin incontinencia de urgencia (3). La diferencia entre ambos géneros se expresa en la severidad de aparición de los síntomas con el aumento de la edad. En estas mujeres la prevalencia de los síntomas de urgencia miccional aumenta del 2 a 19\% después de los 44 años. En cambio, en hombres aumenta desde 0,3 a 8,9\% después de los 64 años. Entonces, la prevalencia de vejiga hiperactiva no difiere entre ambos géneros, sin embargo, las diferencias anatómicas entre hombre y mujer, permiten que con el tiempo, se exprese con síntomas de urgencia miccional, con mayor frecuencia entre mujeres (3). Por otro lado, la vejiga hiperactiva, con o sin incontinencia de urgencia, posee un alto impacto en la calidad de vida, calidad de sueño y salud mental, para ambos géneros (4).

Se estima que las personas con $\mathrm{VH}$, tienen mayores riesgos de salud. Registrando hasta un $20 \%$ de incremento en las visitas a médicos, y hasta un $138 \%$ de incremento en la posibilidad de ingreso a unidades de tratamiento intensivo. Presentan un riesgo mayor de caídas, con resultado de fracturas, generando invalidez y altos costos de salud. Para el año 2004, un estudio de incidencia en Alemania, permitió registrar la morbilidad asociada a VH. Para un total de 6,48 millones de adultos, mayores de 40 años afectados por $\mathrm{VH}$, se encontraron: 310.000 infecciones de piel, 40.000 caídas, 12.000 fracturas y 26.000 casos de depresión (5).

Para el año 2000, en EEUU, se estima que generó gastos de 1,37 billones de dólares en ingresos a UTI, 55 millones en tratamientos de caídas sin fracturas, y 386 millones en caídas con fracturas. Se estima que los costos totales que pueden generar los pacientes con $\mathrm{VH}$, son comparables a los producidos por la osteoporosis y los cánceres ginecológicos y de mama $(1,6)$.

Por todas estas razones, es altamente recomendable, buscar terapias que den una solución efectiva frente a esta patología, que genera altos costos en salud entre quienes la padecen y los diferentes organismos sanitarios $(7,8)$.

Con el objetivo de demostrar la efectividad de la neuromodulación con el sistema de estimulación transcutáneo del nervio tibial posterior, en la inhibición de las contracciones no inhibidas del detrusor, se presenta un caso urge-incontinencia, refractaria a tratamiento tradicional, en que se miden objetivamente los cambios que produce la neuromodulación en la cistometría realizada con urodinamia monocanal.

\section{PACIENTE Y MÉTODO}

Paciente de 45 años, que ingresa a la Unidad de Uroginecología y Cirugía Vaginal de Clínica Las Condes, con el diagnóstico clínico de incontinencia de orina mixta. Presentaba pérdida de orina frente a pequeños esfuerzos, de aumento progresivo, por 10 años. Lo que se objetivó por medio de urodinamia monocanal, en la que la presión de apertura uretral retrógrada obtenida fue de $65 \mathrm{~cm}$ de $\mathrm{H} 2 \mathrm{O}$, por lo que se clasificó como incontinencia de orina de esfuerzo (IOE) tipo II (Figura 1). En la anamnesis se sospechó la presencia de la vejiga hiperactiva con urge-incontinencia, por la frecuencia de más de 10 micciones durante el día y más de 2 durante la noche. El componente de incontinencia de urgencia también fue objetivado mediante urodinamia. Registrándose la presencia de un detrusor hiperactivo, caracterizado por contracciones no inhibidas del detrusor durante la medición cistométrica, durante la fase de llene vesical.

Bajo estos diagnósticos se planificó una cirugía de cinta sub-medio-uretral para dar solución a la 


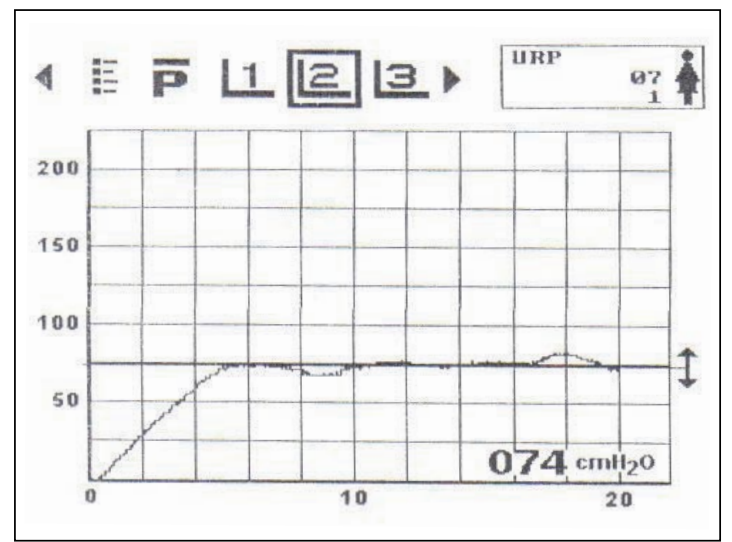

Figura 1. Gráfico correspondiente a la medición URP (presión de retro-resistencia uretral) en la urodinamia monocanal. Valores compatibles con una incontinencia de orina tipo II.

IOE, mediante el sistema TVT-Secur. Esta cinta se colocó en "U", es decir con un ángulo menor de 45 grados, bajo la uretra (9). Durante el postoperatorio, se inició fisioterapia y tratamiento con anticolinérgico (Spasmex), hasta completar una dosis de $45 \mathrm{mg}$ diarios. Sin embargo, al cabo de un mes, sólo se registró una leve mejoría de la urge-incontinencia. Debido a la refractariedad frente a estos tratamientos tradicionales, se decidió realizar neuromodulación, con el sistema de estimulación transcutáneo del nervio tibial posterior.

Técnica de estimulación transcutánea: Se utilizó el equipo BioMed 2000 (BioMedical Life System Inc), con una estimulación con las siguientes características: amplitud de pulso de 200 milisegundos, frecuencia 20 Hertz, y una intensidad regulada hasta lograr la dorsiflexión del ortejo mayor y/o flexión plantar del segundo al quinto ortejo o 10 miliamperes o según la tolerancia de la paciente. La zona de estimulación del electrodo se fijó en la piel, entre 3 a 5 centímetros hacia cefálico, desde la zona medial del maléolo interno. Un segundo electrodo neutro se localizó a nivel medial del calcáneo del pie izquierdo (Figura 2).

Método de medición objetiva de la acción de la neuromodulación transcutánea del nervio tibial posterior sobre el detrusor hiperactivo: Para objetivar la acción de la neuromodulación, sobre las contracciones no inhibidas del detrusor, se realizó primero, una medición cistométrica sin estimulación. Luego se vació la vejiga y se realizó una nueva cistometría, durante estimulación transcutánea del nervio tibial. Durante ambas mediciones cistométricas, se registró la aparición de contracciones no inhibidas del detrusor, la aparición de sensación de urgencia

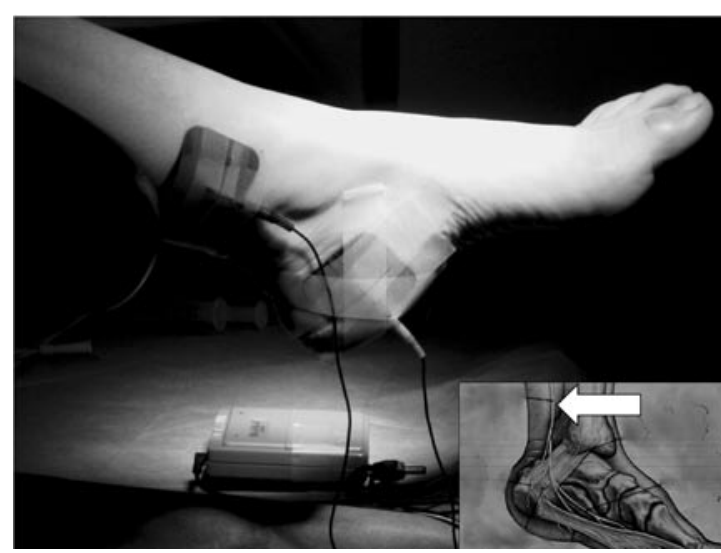

Figura 2. Electrodos situados en la posición de estimulación del nervio tibial posterior. En el recuadro se observa un esquema que muestra el recorrido del nervio tibial posterior (señalado por una flecha). La zona de estimulación del electrodo se fijó en la piel, a 3 a 5 centímetros hacia cefálico, desde la zona medial del maléolo interno, un segundo electrodo neutro se localizó a nivel medial del calcáneo.

miccional y el volumen de la capacidad cistométrica máxima, para luego realizar una comparación de estos parámetros registrados objetivamente mediante los gráficos del cistometrograma.

\section{RESULTADOS}

Las contracciones no inhibidas del detrusor que se observaron durante la fase de llene vesical, disminuyeron notoriamente al realizar la neuromodulación, lo que quedó objetivamente registrado en el gráfico de la cistometría (Figura $3 \mathrm{~A}$ y $3 \mathrm{~B}$ ).

El volumen de la capacidad cistométrica máxima, se vio aumentado al comparar la cistometría durante la neuroestimulación, desde 250 a 350 cc. Por lo que la sensación de llene máximo vesical, se vio desplazada, permitiendo un mayor volumen vesical, antes de sentir un deseo imperioso de orinar. La sensación de urgencia miccional registrada tempranamente durante la cistometría sin neuromodulación, desapareció durante el registro con estimulación transcutánea del nervio tibial.

\section{DISCUSIÓN}

La estimulación transcutánea del nervio tibial es una técnica de neuromodulación mínimamente invasiva y fácil de realizar (10), que incluso se puede utilizar en niños (11). El éxito de esta terapia, se estima que se puede obtener en dos tercios de los 


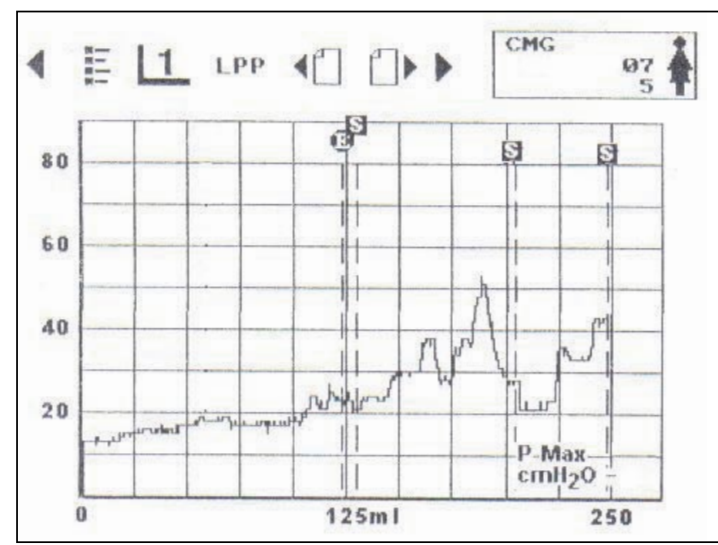

Figura 3A. Gráfico correspondiente a la medición de CMG (cistometrograma) en la urodinamia monocanal que muestra una vejiga hiperactiva.

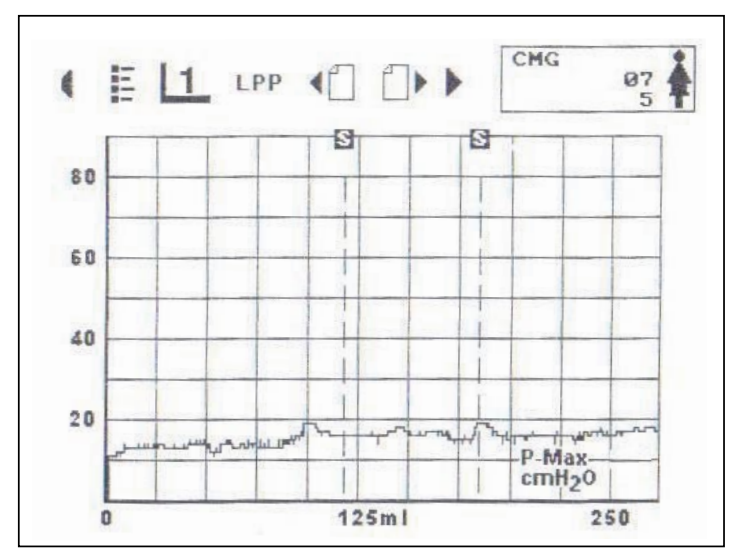

Figura 3B. Registro de CMG (cistometrograma) durante la estimulación del nervio tibial posterior. Se observa la inhibición de la hiperactividad del detrusor.

\section{pacientes (12).}

La neuromodulación periférica entrega una buena alternativa en el tratamiento de pacientes refractarios a terapias tradicionalmente utilizadas en la urge-incontinencia por vejiga hiperactiva, tales como fisioterapia y/o farmacoterapia anticolinérgica. La neuromodulación sacra y la cirugía son tratamientos de segunda línea, invasivos y difíciles de realizar (13).

La neuromodulación periférica es más simple de realizar, tiene escasa morbilidad y es de bajo costo. Sin embargo, este tratamiento se contraindica en pacientes portadores de marcapasos o desfibriladores implantados, pacientes con antecedente de problemas cardíacos, embarazadas o mujeres que pretendan embarazarse durante el tratamiento.
Existe también la posibilidad de realizar una neuromodulación, por medio de electrodos que realizan la estimulación percutáneamente. La ventaja de realizar la estimulación a nivel transcutáneo, es decir por sobre la piel, es que no se requiere la inserción del electrodo, lo que permite otorgar un tratamiento menos invasivo, al no perforar la piel de la paciente. De este modo, lo hace más tolerable y fácil de aplicar al compararlo con la técnica percutánea.

En aquellos casos en que exista una contraindicación o intolerancia a los anticolinérgicos, permitirá disponer de otra terapia no invasiva, que incluso puede combinarse a dosis menores de estos medicamentos, disminuyendo los efectos no deseados (14).

La efectividad y los beneficios de la neuromodulación periférica a través de las diferentes vías y técnicas, en el control de la vejiga hiperactiva son conocidos, sin embargo, durante las últimas tres décadas sólo existen teorías que pretenden explicar su efecto (15-17). A manera de ejemplo, la estimulación de nervio tibial, reduce la expresión de un tercer mensajero conocido como proteína $\mathrm{C}$-fos. Este mensajero se expresa después de la presencia de algún factor irritante químico sobre la vejiga o el tracto urinario bajo. Esto demuestra que la neuromodulación actúa también a nivel de mensajeros (18-20).

El nervio tibial es mixto, posee fibras sensitivas y motoras. La neuromodulación provocada mediante estímulo transcutáneo o percutáneo del tibial, tiene efectos en la vejiga mediante la vía del plexo sacro, a través de estimulación aferente retrógrada (20).

Los resultados obtenidos en esta experiencia clínica, demuestran que los efectos de la neuromodulación transcutánea, son medibles objetivamente en los parámetros urodinámicos, en la urodinamia monocanal. Después de la estimulación, se observó una inhibición de las contracciones no inhibidas del detrusor, y un aumento significativo en el volumen de la capacidad cistométrica máxima. Todo esto se traduce en que la sensación de urgencia miccional, que aparecía tempranamente durante la fase de llene vesical, se ve abolida o retardada en su aparición.

Sin embargo, una sola sesión de estimulación, no es suficiente para mantener estos buenos resultados en el tiempo. Por este motivo, lo recomendable es realizar sesiones de 30 minutos, dos veces por semana, por no menos de un total de 12 sesiones, con la posibilidad de repetir la terapia en caso de recidivar los signos y síntomas de vejiga hiperactiva. Actualmente nos encontramos realizando un seguimiento de pacientes bajo esta terapia, para 
determinar la mantención de los buenos resultados a largo plazo.

Algunos han planteado la posibilidad de considerar a futuro, la implantación en esta zona, de algún dispositivo subcutáneo, que permita realizar estimulación del tibial posterior cuando se requiera (12).

\section{CONCLUSIONES}

La efectividad de la neuromodulación por medio de la estimulación transcutánea del nervio tibial posterior, en la inhibición de las contracciones no inhibidas del detrusor, queda demostrada y objetivada por medio del registro gráfico de la cistometría en la urodinamia monocanal. La estimulación transcutánea de nervio tibial posterior, representa una opción terapéutica en pacientes con urge-incontinencia por vejiga hiperactiva, con todas las características de mínima invasión.

\section{BIBLIOGRAFÍA}

1. Wagner TH, Hu TW, Bentkover J, LeBlanc K, Stewart W, Corey R, Zhou Z, Hunt T. Health-related consequences of overactive bladder. Am J Manag Care 2002;8(19 Suppl):S598-607.

2. Hu TW, Wagner TH. Health-related consequences of overactive bladder: an economic perspective. BJU Int 2005;96 Suppl 1:43-5.

3. Stewart WF, Van Rooyen JB, Cundiff GW, Abrams P, Herzog AR, Corey R, Hunt TL, Wein AJ. Prevalence and burden of overactive bladder in the United States. World J Urol 2003;20(6):327-36.

4. Coyne KS, Payne C, Bhattacharyya SK, Revicki DA, Thompson C, Corey R, Hunt TL. The impact of urinary urgency and frequency on health-related quality of life in overactive bladder: results from a national community survey. Value Health 2004;7(4):455-63.

5. Klotz $T$, Brüggenjürgen $B$, Burkart $M$, Resch $A$. The economic costs of overactive bladder in Germany. Eur Urol 2007;51(6):1654-62.

6. Hu TW, Wagner TH, Bentkover JD, LeBlanc K, Piancentini A, Stewart WF, Corey R, Zhou SZ, Hunt TL. Estimated economic costs of overactive bladder in the United States. Urology 2003;61(6):1123-8.

7. Reeves P, Irwin D, Kelleher C, Milsom I, Kopp Z, Calvert N, Lloyd $\mathrm{A}$. The current and future burden and cost of overactive bladder in five European countries. Eur Urol 2006;50(5):1050-7.
8. Darkow T, Fontes CL, Williamson TE. Costs associated with the management of overactive bladder and related comorbidities. Pharmacotherapy 2005;25(4):5119

9. Solà V, Pardo J, Ricci P. Tercera generación en cirugía mínimamente invasiva para la corrección de la incontinencia de orina de esfuerzo: TVT-Secur. Rev Chil Obstet Ginecol 2007;72(5):304-9

10. Govier FE, Litwiller S, Nitti V, Kreder KJ Jr, Rosenblatt $P$. Percutaneous afferent neuromodulation for the refractory overactive bladder: results of a multicenter study. J Urol 2001;165(4):1193-8

11. De Gennaro M, Capitanucci ML, Mastracci P, Silveri M, Gatti C, Mosiello G. Percutaneous tibial nerve neuromodulation is well tolerated in children and effective for treating refractory vesical dysfunction. J Urol 2004;171(5):1911-3

12. Van Balken MR. Percutaneous tibial nerve stimulation: the Urgent PC device. Expert Rev Med Devices 2007;4(5):693-8

13. Mazurick CA, Landis JR. Evaluation of repeat daily voiding measures in the National Interstitial Cystitis Data Base Study. J Urol 2000;163(4):1208-11.

14. Karademir K, Baykal K, Sen B, Senkul T, Iseri C, Erden D. A peripheric neuromodulation technique for curing detrusor overactivity: Stoller afferent neurostimulation. Scand J Urol Nephrol 2005;39(3):230-3.

15. Van der Pal F, Heesakkers JP, Bemelmans BL. Current opinion on the working mechanisms of neuromodulation in the treatment of lower urinary tract dysfunction. Curr Opin Urol 2006;16(4):261-7.

16. Bemelmans BL, Mundy AR, Craggs MD. Neuromodulation by implant for treating lower urinary tract symptoms and dysfunction. Eur Urol 1999;36(2):8191.

17. Fall M, Lindström S. Electrical stimulation. A physiologic approach to the treatment of urinary incontinence. Urol Clin North Am 1991;18(2):393-407.

18. Chang CJ, Huang ST, Hsu K, Lin A, Stoller ML, Lue TF. Electroacupuncture decreases c-fos expression in the spinal cord induced by noxious stimulation of the rat bladder. J Urol 1998;160(6 Pt 1):2274-9.

19. Birder LA, Roppolo JR, Erickson VL, de Groat WC. Increased c-fos expression in spinal lumbosacral projection neurons and preganglionic neurons after irritation of the lower urinary tract in the rat. Brain Res 1999;834(1-2):55-65.

20.Ishigooka M, Nakada T, Hashimoto T, Zermann $\mathrm{DH}$, Schmidt RA. c-Fos expression in the spinal cord after acute sacral segmental nerve stimulation. Neurourol Urodyn 2002;21(5):495-501. 